\title{
A Modified Comprehensive Model for Piezoelectric Stack Actuators and Corresponding Parameter Identification Method
}

\author{
Haigen Yang ${ }^{1}$ and Wei $\mathrm{Zhu}^{2}$ \\ ${ }^{1}$ Nanjing University of Posts and Telecommunications, Nanjing 210003, China \\ ${ }^{2}$ Institute of Launch Dynamics, Nanjing University of Science and Technology, Nanjing 210094, China \\ Correspondence should be addressed to Haigen Yang; zhuweicqu@163.com
}

Received 9 August 2015; Accepted 20 October 2015

Academic Editor: Giovanni Berselli

Copyright @ $2015 \mathrm{H}$. Yang and W. Zhu. This is an open access article distributed under the Creative Commons Attribution License, which permits unrestricted use, distribution, and reproduction in any medium, provided the original work is properly cited.

\begin{abstract}
In order to accurately model the hysteresis and dynamic characteristics of piezoelectric stack actuators (PSAs), consider that a linear force and a hysteresis force will be generated by piezoelectric wafers under the voltage applied to a PSA, and the total force suffering from creep will result in the forced vibration of the two-degree-of-freedom mass-spring-damper system composed of the equivalent mass, stiffness, and damping of the piezoelectric wafers and the bonding layers. A modified comprehensive model for PSAs is put forward by using a linear function, an asymmetrical Bouc-Wen hysteresis operator, and a creep function to model the linear force, the hysteresis force, and the creep characteristics, respectively. In this way, the effect of the bonding layers on the hysteresis and dynamic characteristics of PSAs can be analyzed via the modified comprehensive model. The experimental results show that the modified comprehensive model for PSAs with the corresponding parameter identification method can accurately portray the hysteresis and dynamic characteristics of PSAs fabricated by different layering/stacking processes. Finally, the theoretical analyzing on utilizing the modified comprehensive model to linearize the hysteresis characteristics and design the dynamic characteristics of PSAs is given.
\end{abstract}

\section{Introduction}

In the two decades, piezoelectric actuators have been widely used in many smart structures [1-3]. However, the displacement output of single-wafer piezoelectric actuators is relatively small, and piezoelectric stack actuators (PSAs) are an alternative to increase the displacement output with high resolution and high positioning accuracy. PSAs are realized by layering/stacking multichip piezoelectric wafers in series mechanically and connecting electrodes in parallel electrically. The layering/stacking processes of multichip piezoelectric wafers can be categorized as three types: gluing piezoelectric wafers and electrodes, sintering piezoelectric wafers and electrodes layer by layer, and direct sintering piezoelectric wafers and electrodes as a whole (the cofired technology).

In order to explain the influence of layering/stacking processes on the properties of PSAs, provide reference for designing PSAs with high-performance, and effectively and accurately control the displacement output of PSAs, it is very meaningful to accurately model the hysteresis and dynamic characteristics of PSAs.

However, the current literatures indicated that the existing researches are mainly focused on hysteresis models for PSAs, such as the Preisach model [4-7], the Prandtl-Ishlinskii model $[8,9]$, the Polynomial model $[10,11]$, the Neural Network model $[12,13]$, the Maxwell model $[14,15]$, the Duhem model [16], the LuGre model [17, 18], the differential model [19], and the Bouc-Wen model [19-21]. These hysteresis models for PSAs can only simulate the hysteresis characteristics of PSAs. When simulating the dynamic characteristics of a PSA, the PSA is generally considered as a single-degreeof-freedom (DOF) mass-spring-damper system composed of the equivalent mass, damping, and stiffness of the PSA $[17,18,22,23]$. However, due to ignoring the bonding layers, this method cannot reflect the properties differences of PSAs with different layering/stacking processes. 


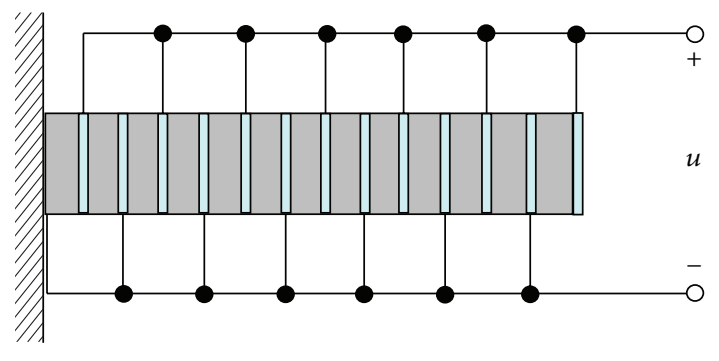

Piezoelectric wafer

\Electrode and bonding layer

FIGURE 1: Schematic representation of PSAs.

In this paper, a modified comprehensive model for PSAs and a corresponding parameter identification method will be put forward and experimentally verified. In Section 2, consider that a linear force and a hysteresis force will be generated by piezoelectric wafers under the applied voltage to a PSA, and the total force suffering from creep will result in the forced vibration of the two-degree-of-freedom (2-DOF) mass-spring-damper system composed of the equivalent mass, stiffness, and damping of the piezoelectric wafers and the bonding layers. A modified comprehensive model for PSAs is put forward by using a linear function, an asymmetrical Bouc-Wen hysteresis operator, and a creep function to model the linear force, the hysteresis force, and the creep characteristics, respectively. In Section 3, through separating the linear component of the proposed modified comprehensive model from the hysteresis component, the parameter identification method is established through identifying the parameters for the linear and hysteresis components separately by the limit theorem of Laplace transformation and the least-squares method, respectively. In Section 4, the performance of the proposed modified comprehensive model with the corresponding parameter identification method is experimentally verified by the established experimental setup. In Section 5, the applications of the modified comprehensive model are discussed. In Section 6, some conclusions are drawn.

\section{Modified Comprehensive Model for PSAs}

Figure 1 shows the schematic representation of PSAs. Observing Figure 1, a PSA is composed of piezoelectric wafers, electrodes, and bonding layers, the multichip piezoelectric wafers are layered/stacked by the bonding layers in series mechanically, and the electrodes are connected in parallel electrically. Because the mechanical properties of the piezoelectric wafers are similar to those of the electrodes, they can be considered as a whole, when modeling the dynamic characteristics of a PSA.

Consider that a linear force and a hysteresis force will be generated by piezoelectric wafers under the applied voltage

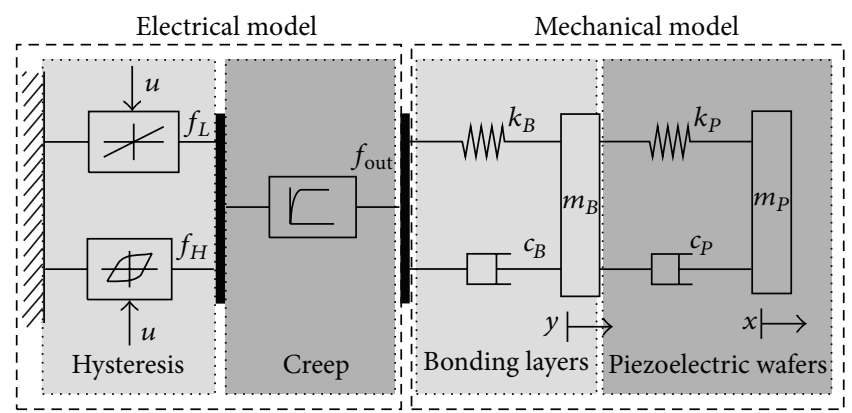

Figure 2: Modified comprehensive model for PSAs.

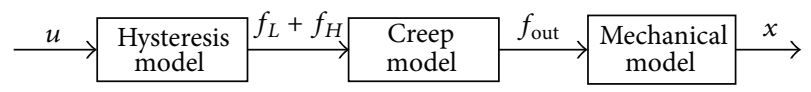

FIGURE 3: Block diagram of the modified comprehensive model for PSAs.

to a PSA, and the total force, which has suffered from creep, will result in the forced vibration of the 2-DOF mass-springdamper system composed of the equivalent mass, stiffness, and damping of the piezoelectric wafers and the bonding layers. On this basis, the modified comprehensive model for PSAs is shown in Figure 2 and can be expressed as

$$
\begin{aligned}
f_{L} & =\mathbf{L}(u) \\
f_{H} & =\mathbf{H}(u) \\
f_{\text {out }} & =\mathbf{C}\left(f_{L}+f_{H}\right) \\
f_{\text {out }} & =m_{P} \ddot{y}+c_{P} \dot{y}+k_{P} y \\
c_{P} \dot{y}+k_{P} y & =m_{B} \ddot{x}+c_{B}(\dot{x}-\dot{y})+k_{B}(x-y),
\end{aligned}
$$

where $f_{L}(t)$ and $f_{H}(t)$ are the linear and hysteresis forces generated by the PSA, respectively; $\mathbf{L}(\cdot)$ and $\mathbf{H}(\cdot)$ are the linear and hysteresis functions to model the linear and hysteresis forces generated by the PSA with the applied voltage, respectively; $f_{\text {out }}$ is the total force; $\mathbf{C}(\cdot)$ is the creep function to model the creep characteristics; $m_{P}, c_{P}$, and $k_{P}$ are the equivalent mass, damping, and stiffness of the piezoelectric wafers, respectively; $y(t)$ is the displacement output of the bonding layers; $\dot{y}(t)$ and $\ddot{y}(t)$ are the first and second order derivatives of $y(t)$ with respect to time, respectively; $m_{B}$, $c_{B}$, and $k_{B}$ are the equivalent mass, damping, and stiffness of the bonding layers, respectively; $x(t)$ is the displacement output of the PSA; $\dot{x}(t)$ and $\ddot{x}(t)$ are the first and second order derivatives of $x(t)$ with respect to time, respectively.

According to (1)-(5) and Figure 2, the modified comprehensive model for PSAs can be considered as an electric model in series with a mechanical model, and the electric model can be considered as a hysteresis model in series with a creep model, as shown in Figure 3.

In Figure 3, the hysteresis model, which is given by (1) and (2), is used to model the linear and hysteresis forces generated by the PSA with the applied voltage; the creep model, which is given by (3), is used to model the creep characteristics; 
the mechanical model, which is given by (4) and (5), is used to model the displacement output that resulted from the linear and hysteresis forces generated by the PSA. According to (4) and (5), the mechanical model can be equivalent to the 2-DOF mass-spring-damper system composed of the equivalent mass, stiffness, and damping of the piezoelectric wafers and the bonding layers.

Ignoring the bonding layers yields the simplified comprehensive model, and the mechanical model of the simplified comprehensive model can be expressed as

$$
f_{\text {out }}(t)=\left(m_{P}+m_{B}\right) \ddot{x}(t)+\bar{c}_{P} \dot{x}(t)+\bar{k}_{P} x(t),
$$

where $\bar{c}_{P}$ and $\bar{k}_{P}$ are the equivalent damping and stiffness of PSAs, respectively, and can be obtained by the identification method proposed in [20]. Equations (1)-(3) and (6) define the simplified comprehensive model for PSAs. The mechanical model of the simplified comprehensive model is equivalent to the single-DOF mass-spring-damper system composed of the equivalent mass, stiffness, and damping of the PSA.

2.1. Electrical Model. The linear function, given by (1), models the linear force and can be expressed as

$$
f_{L}=\mathbf{L}(u)=k_{L} u+f_{0},
$$

where $k_{L}$ is the ratio between the linear force and the voltage applied to PSAs and $f_{0}$ is the initial force. In the zero initial condition, $f_{0}=0$.

The hysteresis function of the electric model given by (2) simulates the hysteresis force generated by PSAs under the applied voltage. Considering that the hysteresis of PSAs is asymmetrical [3], utilizing the asymmetrical Bouc-Wen hysteresis operator to simulate the hysteresis force yields

$$
\begin{aligned}
\dot{f}_{H} & =\dot{\mathbf{H}}(u) \\
& =A \dot{u}-\beta|\dot{u}|\left|f_{H}\right|^{n-1} f_{H}-\gamma \dot{u}\left|f_{H}\right|^{n}+\delta u \operatorname{sgn}\left(f_{H}\right),
\end{aligned}
$$

where $A, \beta, \gamma$, and $n$ are the parameters of the asymmetrical Bouc-Wen hysteresis operator; $\operatorname{sgn}(x)=1, x>0 ;-1, x<$ 0 ; $\delta$ is the asymmetrical factor, and $\delta<0$ when modeling PSAs.

The creep model, which is given by (3), is used to model the creep characteristics and can be expressed as

$$
f_{\text {out }}=\left(1-e^{-t / \tau}\right)\left(f_{L}+f_{H}\right)
$$

where $\tau$ is the time constant of the creep characteristics.

According to the electric model given by (7)-(9), the bonding layers do not affect the hysteresis characteristics of PSAs.

2.2. Mechanical Model. According to (4) and (5), the transfer function of the mechanical model can be expressed as

$$
\begin{aligned}
& \frac{x(s)}{f_{L}(s)+f_{H}(s)} \\
& =\frac{\left(c_{B}+c_{P}\right) s+\left(k_{B}+k_{P}\right)}{\left(m_{B} s^{2}+c_{B} s+k_{B}\right)\left(m_{P} s^{2}+c_{P} s+k_{P}\right)} .
\end{aligned}
$$

According to (10), the transfer function has one zero and four poles. The zero can be expressed as

$$
z_{1}=-\frac{k_{B}+k_{P}}{c_{B}+c_{P}}
$$

The four poles can be expressed as

$$
\begin{aligned}
& s_{1,2}=-\frac{c_{P}}{m_{P}} \pm \frac{1}{m_{P}} \sqrt{c_{P}^{2}-4 m_{P} k_{P}} \\
& s_{3,4}=-\frac{c_{B}}{m_{B}} \pm \frac{1}{m_{B}} \sqrt{c_{B}^{2}-4 m_{P} k_{B}}
\end{aligned}
$$

According to (12), all of the four poles have negative real parts, which indicate that the mechanical model is stable. According to (10)-(12), the mechanical characteristics of the bonding layers affect the zero $z_{1}$ and poles $s_{3,4}$. Therefore, if the mechanical characteristics of the bonding layers are changed, the dynamic performance of the PSA will be changed correspondingly.

\section{Parameter Identification Method}

In order to identify the parameters of the modified comprehensive model for PSAs, the linear component of the modified comprehensive model is separated from the hysteresis component. The parameters for the linear and hysteresis components are separately identified by utilizing the limiting theorem of the Laplace transformation and the least-squares method, respectively.

3.1. Parameter Identification Method of the Linear Component. When the applied voltage is small, the PSA can be approximated to a linear system and $f_{H}=0$ [20]. According to (7)(11), the transfer function of the linear comprehensive model can be rewritten as

$$
\frac{x(s)}{u(s)}=\frac{k_{L}}{\tau s+1} \frac{\left(c_{B}+c_{P}\right) s+\left(k_{B}+k_{P}\right)}{\left(m_{B} s^{2}+c_{B} s+k_{B}\right)\left(m_{P} s^{2}+c_{P} s+k_{P}\right)} .
$$

According to (13), the steady-state output of the unit step response is given by

$$
x_{s}=\frac{k_{L}\left(k_{B}+k_{P}\right)}{k_{B} k_{P}} \text {. }
$$

According to (13) and (14), the normalized unit transfer function can be expressed as

$$
\bar{G}(s)=\frac{b_{1} s+1}{a_{5} s^{5}+a_{4} s^{4}+a_{3} s^{3}+a_{2} s^{2}+a_{1} s+1},
$$

where $\bar{G}(s)$ is the normalized transfer function; $a_{5}, a_{4}, a_{3}, a_{2}$, $a_{1}$, and $b_{1}$ are the undetermined parameters with values larger than zero. Let $g(t)$ be the Laplace inverse transform of $\bar{G}(s)$. 
Define

$$
g_{1}(t)=\int_{0}^{t}[1-g(\tau)] \mathrm{d} \tau
$$

According to the limiting theorem of the Laplace transformation, we have

$$
\bar{G}_{1}(s)=L\left[g_{1}(t)\right]=\frac{1}{s^{2}}[1-\bar{G}(s)] .
$$

According to (15) and (17), we have

$$
K_{1}=\lim _{t \rightarrow \infty} g_{1}(t)=\lim _{s \rightarrow 0} \bar{G}_{1}(s)=a_{1}-b_{1} .
$$

And so forth, let

$$
g_{i}(t)=\int_{0}^{t}\left[K_{i-1}-g_{i-1}(\tau)\right] \mathrm{d} \tau \quad(i=2,3,4,5,6) .
$$

We have

$$
\begin{aligned}
K_{i} & =\lim _{t \rightarrow \infty} g_{i}(t)=\lim _{s \rightarrow 0} \bar{G}_{i}(s) \\
& =(-1)^{i} b_{i}+K_{i-1} a_{1}-K_{i-2} a_{2}+\cdots+(-1)^{i-1} a_{i},
\end{aligned}
$$

where $b_{i}=0$, when $i>2$. A set of equations can be obtained as

$$
\begin{gathered}
K_{1}=a_{1}-b_{1} \\
K_{2}=K_{1} a_{1}-a_{2} \\
\vdots \\
K_{6}=K_{5} a_{1}-K_{4} a_{2}+\cdots+\left(-a_{5}\right) .
\end{gathered}
$$

According to (21), the parameters $a_{5}, a_{4}, a_{3}, a_{2}, a_{1}$, and $b_{1}$ can be identified when the normalized unit step response of the PSA is accessed.

\subsection{Parameter Identification Method of the Nonlinear Com-} ponent. When the frequency of the applied voltage to a PSA is less than $1 \mathrm{~Hz}$, PSAs can be considered as the quasi-static condition, and the change of the displacement output of PSAs is slow. So the velocity term and the acceleration term in (4) and (5) are small and can be ignored. At the same time, the impact of the creep characteristics on PSAs is also very small. Therefore, the quasi-static model of PSAs can be expressed as

$$
\begin{aligned}
u+\left(\frac{f_{H}+f_{0}}{k_{L}}\right)= & \frac{k_{B} k_{P}}{k_{L}\left(k_{B}+k_{P}\right)} x \\
\dot{f}_{H}= & A \dot{u}-\beta|\dot{u}|\left|f_{H}\right|^{n-1} f_{H}-\gamma \dot{u}\left|f_{H}\right|^{n} \\
& +\delta u \operatorname{sgn}\left(f_{H}\right) .
\end{aligned}
$$

According to (15), let

$$
k=\frac{k_{L}\left(k_{B}+k_{P}\right)}{k_{B} k_{P}}=x_{s},
$$

where $k$ is the steady-state output of the unit step response of the PSA and can be identified when the unit step response of the PSA is accessed.

The state of the PSA without any applied voltage can be considered as the zero initial condition. According to the inverse piezoelectric effect, the PSA will return to the zero initial condition when the applied voltage disappears for an enough time, which is different for different PSAs. Applying the voltage $u(t)$ with frequency of less than $1 \mathrm{~Hz}$ to the PSA under the zero initial condition, we have

$$
f_{0}=0
$$

Let $h=\left(k_{B} k_{P} /\left(k_{B}+k_{P}\right)\right) f_{H}$. According to (22) and (24), the following equations can be obtained:

$$
\begin{aligned}
x(t)= & k u(t)+h(t) \\
\dot{h}(t)= & A \dot{u}(t)-\beta|\dot{u}(t)||h(t)|^{n-1} h(t)-\gamma \dot{u}(t)|h(t)|^{n} \\
& +\delta u(t) \operatorname{sgn}(h(t)) .
\end{aligned}
$$

Let $h(t)=0$. From (25), we have

$$
x(t)-k u(t)=0 .
$$

Assume that $\left(u_{+}, x_{+}\right)$and $\left(u_{-}, x_{-}\right)$are the solutions of (27) when $\dot{u}(t)>0$ and $\dot{u}(t)<0$ in the $i$ th $\left(i=1,2, \ldots, N_{1}\right)$ period, respectively. According to (25) and (26), we have

$$
\begin{aligned}
& \dot{x}_{+}=\left(A_{i}+k_{v}\right) \dot{u}_{+}+\delta_{i} u_{+} \\
& \dot{x}_{-}=\left(A_{i}+k_{v}\right) \dot{u}_{-}-\delta_{i} u_{-}
\end{aligned}
$$

where $A_{i}$ and $\delta_{i}$ represent the values of $A$ and $\delta$ identified with the experimental data in the $i$ th period, respectively. According to (28), we can get

$$
\begin{aligned}
A_{i} & =\frac{\dot{x}_{+} u_{-}+\dot{x}_{-} u_{+}}{\dot{u}_{+} u_{-}+\dot{u}_{-} u_{+}}-k_{v} \\
\delta_{i} & =\frac{\dot{x}_{+} \dot{u}_{-}-\dot{x}_{-} \dot{u}_{+}}{\dot{u}_{+} u_{-}+\dot{u}_{-} u_{+}} .
\end{aligned}
$$

According to (29) and the least-squares method, the parameters $A$ and $\delta$ can be given by

$$
\begin{gathered}
A=\frac{1}{N_{1}} \sum_{i=1}^{N_{1}} A_{i} \\
\delta=\frac{1}{N_{1}} \sum_{i=1}^{N_{1}} \delta_{i} .
\end{gathered}
$$




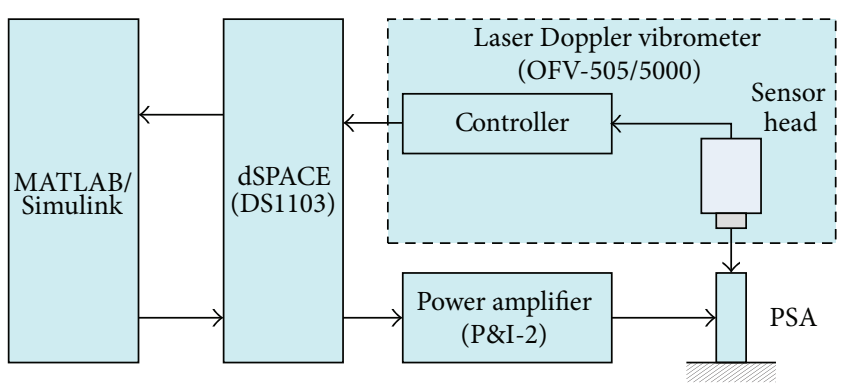

(a)

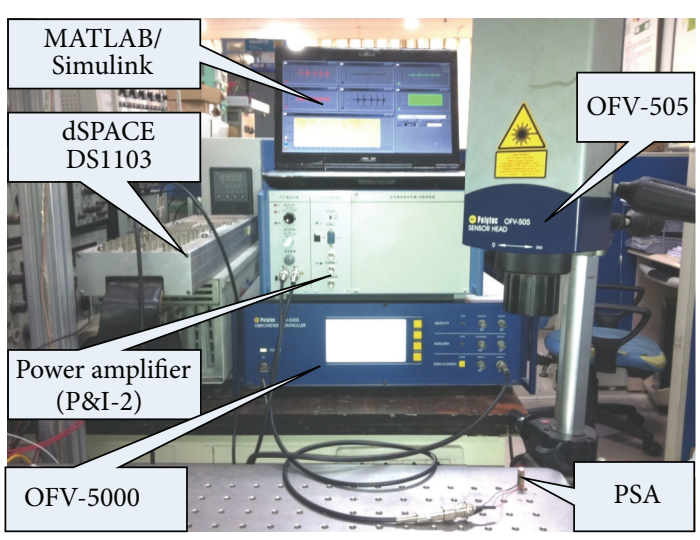

(b)

FIgURE 4: Experimental setup for PSAs: (a) the schematic and (b) the photograph.

When $\dot{u}(t)>0$, consider a set of points $u_{1}, \ldots, u_{i}, \ldots, u_{N_{2}}$ $\left(i=1,2, \ldots, N_{2}\right)$ in $u(t)$, which ensures that the corresponding hysteresis displacements $h_{1}, \ldots, h_{i}, \ldots, h_{N_{2}}$ are larger than zero. Equation (26) can be rewritten as

$$
\frac{\dot{h}_{1}-\delta u_{1}}{\dot{u}_{1}}=A-(\beta+\gamma) h_{1}^{n}
$$

$$
\frac{\dot{h}_{N_{2}}-\delta u_{N_{2}}}{\dot{u}_{N_{2}}}=A-(\beta+\gamma) h_{N_{2}}^{n} .
$$

Let $\beta+\gamma>0$. According to (31) and the least-squares method, we have

$$
\begin{aligned}
n & =\frac{N_{2} \sum_{i=1}^{N_{2}}\left[\ln h_{i} \ln \left(A-\left(\dot{h}_{i}-\delta u_{i}\right) / \dot{u}_{i}\right)\right]-\sum_{i=1}^{N_{2}} \ln h_{i} \sum_{i=1}^{N_{2}} \ln \left(A-\left(\dot{h}_{i}-\delta u_{i}\right) / \dot{u}_{i}\right)}{N_{2} \sum_{i=1}^{N_{2}}\left(\ln h_{i}\right)^{2}-\left(\sum_{i=1}^{N_{2}} \ln h_{i}\right)^{2}} \\
\beta+\gamma & =e^{\left(\sum_{i=1}^{N_{2}} \ln \left(A-\left(\dot{h}_{i}-\delta u_{i}\right) / \dot{u}_{i}\right)-n \sum_{i=1}^{N_{2}} \ln h_{i}\right) / N_{2}} .
\end{aligned}
$$

When $\dot{u}(t)<0$, consider a set of points $u_{N_{2}+1}, \ldots, u_{N_{2}+i}$, $\ldots, u_{2 N_{2}}\left(i=1,2, \ldots, N_{2}\right)$ in $u(t)$, which ensures that the corresponding hysteresis displacements $h_{\mathrm{N}_{2}+1}, \ldots, h_{\mathrm{N}_{2}+i}, \ldots$, $h_{2 N_{2}}$ are larger than zero. Let $-\beta+\gamma>0$. We have

$$
-\beta+\gamma=e^{\left(\sum_{i=1}^{N_{2}} \ln \left(A-\left(\dot{h}_{N_{2}+i}+\delta u_{N_{2}+i}\right) / \dot{u}_{N_{2}+i}\right)-n \sum_{i=1}^{N_{2}} \ln h_{N_{2}+i}\right) / N_{2}} .
$$

According to (33) and (34), the parameters $\beta$ and $\gamma$ can be given by

$$
\begin{aligned}
\beta= & \frac{1}{2}\left[e^{\left(\sum_{i=1}^{N_{2}} \ln \left(A-\left(\dot{h}_{i}-\delta u_{i}\right) / \dot{u}_{i}\right)-n \sum_{i=1}^{N_{2}} \ln h_{i}\right) / N_{2}}\right. \\
& \left.-e^{\left(\sum_{i=1}^{N_{2}} \ln \left(A-\left(\dot{h}_{N_{2}+i}+\delta u_{N_{2}+i}\right) / \dot{u}_{N_{2}+i}\right)-n \sum_{i=1}^{N_{2}} \ln h_{N_{2}+i}\right) / N_{2}}\right] \\
\gamma= & \frac{1}{2}\left[e^{\left(\sum_{i=1}^{N_{2}} \ln \left(A-\left(\dot{h}_{i}-\delta u_{i}\right) / \dot{u}_{i}\right)-n \sum_{i=1}^{N_{2}} \ln h_{i}\right) / N_{2}}\right. \\
& \left.+e^{\left(\sum_{i=1}^{N_{2}} \ln \left(A-\left(\dot{h}_{N_{2}+i}+\delta u_{N_{2}+i}\right) / \dot{u}_{N_{2}+i}\right)-n \sum_{i=1}^{N_{2}} \ln h_{N_{2}+i}\right) / N_{2}}\right] .
\end{aligned}
$$

According to (30), (32), and (35), the parameters $A, \delta, n$, $\beta$, and $\gamma$ for the proposed modified comprehensive model can be identified if the displacement output $x(t)$ of the PSA with the applied voltage $u(t)$ is accessed.

\section{Experimental Verification and Analysis}

4.1. Experimental Setup. In order to experimentally validate the proposed modified comprehensive model for PSAs and the corresponding parameter identification method, the schematic and photograph of the experimental setup are shown in Figures 4(a) and 4(b), respectively. According to Figure 4, the experimental setup is composed of the power amplifier for piezoelectric actuators (type: P\&I-2, peak power: $320 \mathrm{~W}$ ) [7], laser Doppler vibrometer (LDV, type: OFV-505/5000, Polytec, measurement range: $-40 \sim+40 \mu \mathrm{m}$, resolution: $2 \mathrm{~nm}$ ), and real-time simulation system (dSPACE 


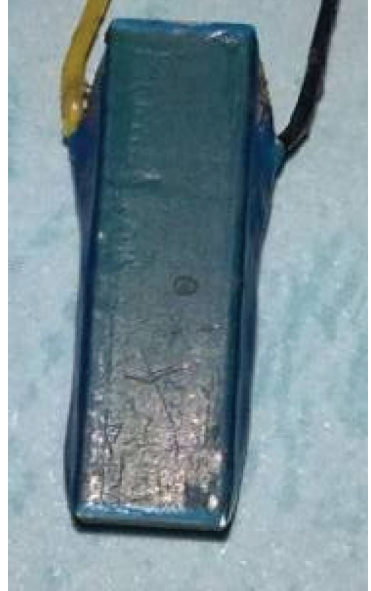

(a)

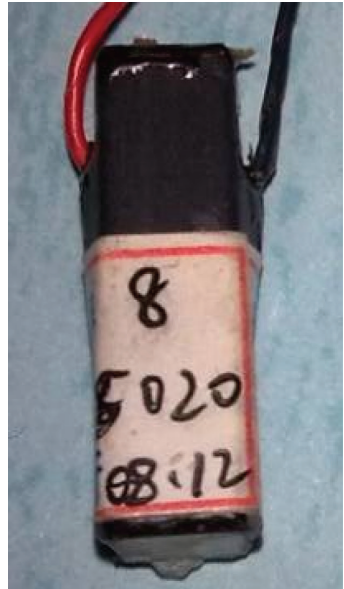

(b)

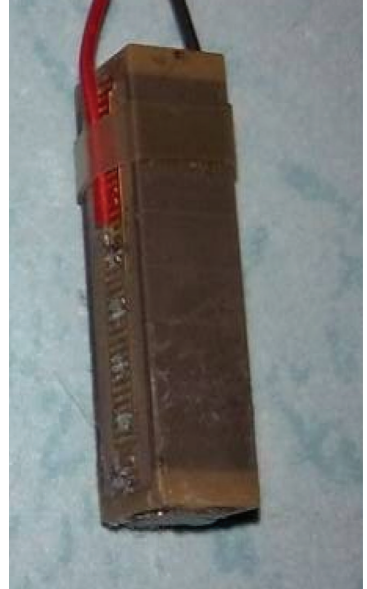

(c)

FIGURE 5: Photographs of the PSAs fabricated by (a) gluing piezoelectric wafers and electrodes (type: PTBS200), (b) sintering piezoelectric ceramic wafers and electrodes layer by layer (type: WYDS0808020), and (c) direct sintering piezoelectric ceramic wafers and electrodes as a whole (the cofired technology) (type: P885.51).

DS1103 with MATLAB/Simulink). When conducting experiments, PSAs, whose displacement outputs are monitored by the LDV, are driven by the power amplifier. The applied voltage to the power amplifier and the output from the LDV are acquired to the host computer by the real-time simulation system.

\subsection{Hysteresis and Dynamic Characteristics of Three PSAs} Fabricated by Different Layering/Stacking Processes. Three PSAs fabricated by different layering/stacking processes are tested by the established experimental setup. Figures 5(a), 5(b), and 5(c) show the photographs of the PSAs fabricated by gluing piezoelectric wafers and electrodes (type: PTBS200, size: $5 \times 5 \times 18 \mathrm{~mm}$, applied voltage: $0-200 \mathrm{~V}$, output displacement: $0-18 \mu \mathrm{m}$, equivalent capacitance: $1.6 \mu \mathrm{F}$, Kunshan $\mathrm{GmbH}$, China), sintering piezoelectric wafers and electrodes layer by layer (type: WYDS0808020, size: $5 \times$ $5 \times 18 \mathrm{~mm}$, applied voltage: $0-200 \mathrm{~V}$, output displacement: $0-18 \mu \mathrm{m}$, equivalent capacitance: $1.6 \mu \mathrm{F}$, China Electronic Technology Group Corporation 26th Research Institute), and directly sintering piezoelectric wafers and electrodes as a whole using the cofired technology (type: P885.51, size: $5 \times$ $5 \times 18 \mathrm{~mm}$, applied voltage: $0-120 \mathrm{~V}$, output displacement: $0-$ $18 \mu \mathrm{m}$, equivalent capacitance: $1.5 \mu \mathrm{F}$, the Physik Instrumente $\mathrm{GmbH}$, Germany), respectively. The rise times of the applied voltages of three PSAs, which jump up from 0 to $100 \mathrm{~V}$, are less than $30 \mu \mathrm{s}$, when the peak voltage of the power amplifier is $320 \mathrm{~W}$ [7].

The running frequency of PSAs is restricted by the heat dissipation, the production process, and the equivalent capacitive load to some extent. So the step responses of PSAs are used to evaluate their dynamic performance. The measured hysteresis curves and unit step responses of three PSAs, as shown in Figure 5, are shown in Figures 6 and 7, respectively. The unit step responses of PSAs in this paper

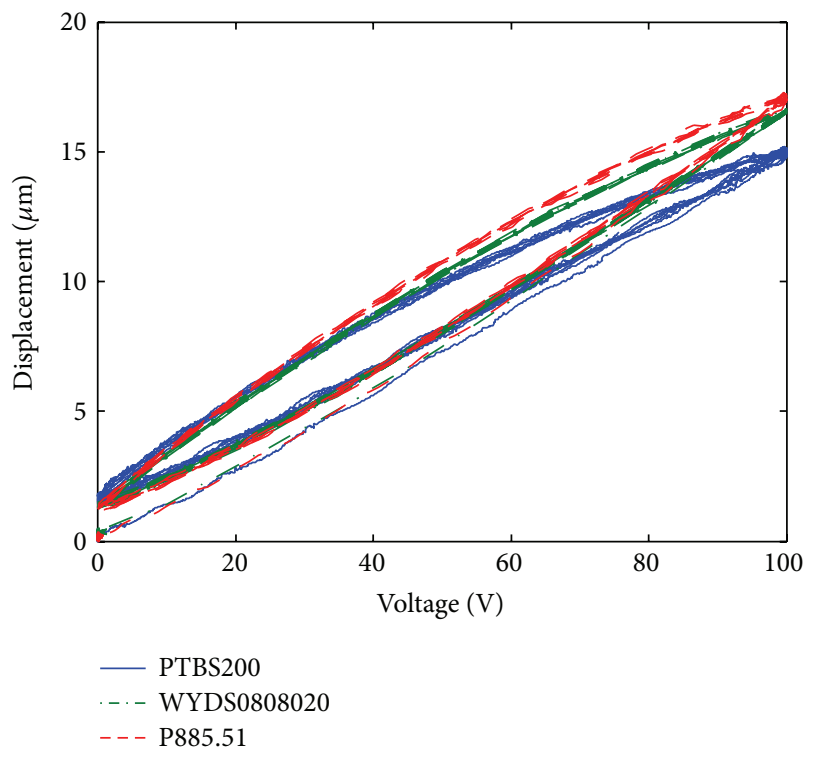

FIGURE 6: Measured hysteresis curves of the PSAs fabricated by different layering/stacking processes.

have been normalized to compare with each other by the normalized formula expressed as

$$
\bar{X}(t)=\frac{X(t)}{X_{s}},
$$

where $X(t)$ is the unit step response; $X_{s}$ is the steady-state output of the unit step response; $\bar{X}(t)$ is the normalized unit step response. 
TABLE 1: Identified parameters of the modified comprehensive model.

\begin{tabular}{lcccccc}
\hline Types & $b_{1}$ & $a_{5}$ & $a_{4}$ & $a_{3}$ & $a_{2}$ \\
\hline PTBS200 & $1.2339 * 10^{-13}$ & $4.113 * 10^{-23}$ & $2.742 * 10^{-18}$ & $1.2339 * 10^{-13}$ & $4.5243 * 10^{-9}$ & $1.0537 * 10^{-4}$ \\
WYDS0808020 & $1.1254 * 10^{-13}$ & $2.6171 * 10^{-22}$ & $2.6172 * 10^{-18}$ & $1.1254 * 10^{-13}$ & $7.4692 * 10^{-9}$ & $1.9012 * 10^{-4}$ \\
P885.51 & $1.1697 * 10^{-13}$ & $7.6782 * 10^{-22}$ & $2.5994 * 10^{-18}$ & $1.1697 * 10^{-13}$ & $6.6285 * 10^{-9}$ & $1.2329 * 10^{-4}$ \\
\hline & $k(\mu \mathrm{m} / \mathrm{V})$ & $A$ & $\beta$ & $n$ & $\gamma$ \\
\hline PTBS200 & 0.1705 & -0.0695 & 0.0155 & 1.205 & -0.0196 & 0.0085 \\
WYDS0808020 & 0.1812 & -0.0705 & 0.0215 & 1.51 & -0.0225 & 0.004 \\
P885.51 & 0.1858 & -0.0724 & 0.0234 & 1.52 & -0.025 & 0.0075 \\
\hline
\end{tabular}

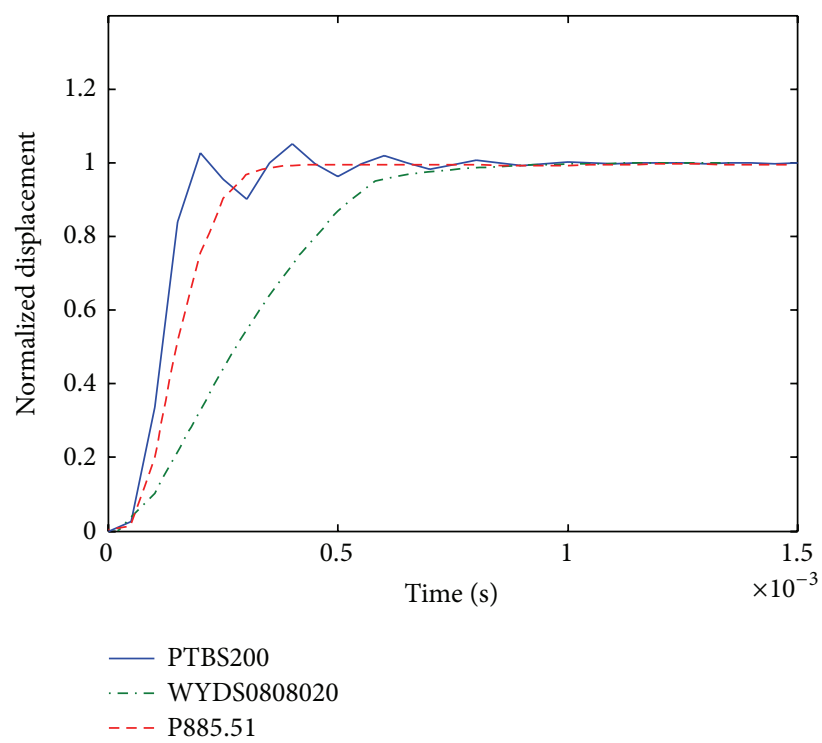

FIGURE 7: Measured normalized unit step responses of the PSAs fabricated by different layering/stacking processes.

Observing Figure 6, the hysteresis characteristics of the three PSAs are similar. But observing Figure 7, the step responses of the three PSAs are apparently different. PTBS200 is an underdamping system, but both WYDS0808020 and P885.51 are overdamping systems. PTBS200 maybe possesses the shortest life time due to the overshot of the underdamping system and P885.51 possesses the shortest settling time due to the overdamping systems with appropriate damping. Therefore, the layering/stacking processes do not affect the hysteresis characteristics but affect the dynamic characteristics, which are consistent with the theoretical analysis presented in Section 2.

4.3. Parameter Identification. According to (21), (23), (30), (33), (37), and (38) and a set of data as shown in Figures 6 and 7 , the identified parameters of the modified comprehensive model for PTBS200, WYDS0808020, and P885.51 are listed in Table 1.
4.4. Experimental Verification. The hysteresis and dynamic characteristics of the three PSAs can be modeled by the proposed modified comprehensive model given by Table 1 .

The hysteresis curves from the three PSAs measured by the LDV and predicted by the modified comprehensive model are shown in Figures 8(a), 8(b), and 8(c), respectively. The corresponding errors between the predicted and measured displacement outputs are shown in Figure 8(d). From Figure 8, the maximum absolute error is $0.41 \mu \mathrm{m}$ and the maximum relative error is $2.65 \%$, so the modified comprehensive model with the correspondingly identified parameters can portray the hysteresis characteristics well. The normalized unit step responses of the three PSAs measured by the LDV and predicted by the modified comprehensive model are shown in Figure 9(a). The corresponding errors between the predicted and measured normalized unit step responses are shown in Figure 9(b). As it can be observed in Figure 9, the maximum absolute error is 0.0142 and the maximum relative error is $1.42 \%$; therefore, the modified comprehensive model with the correspondingly identified parameters can also accurately model the dynamic characteristics.

Figure 10 shows the measured and modeled responses of P885.51 under a nonperiodic voltage. The corresponding time history of the modeling output displacement error is also shown in Figure 10. It can be observed in Figure 10 that the modified comprehensive model can accurately model the characteristics of the PSAs under applied voltages with various frequencies. In summary, the proposed modified comprehensive model along with the corresponding parameter identification method can accurately portray the hysteresis and dynamic characteristics of PSAs fabricated by different layering/stacking processes.

\section{Potential Applications of the Modified Comprehensive Model}

5.1. Linearizing the Hysteresis Characteristics. Because the hysteresis force of PSAs cannot be directly measured by 


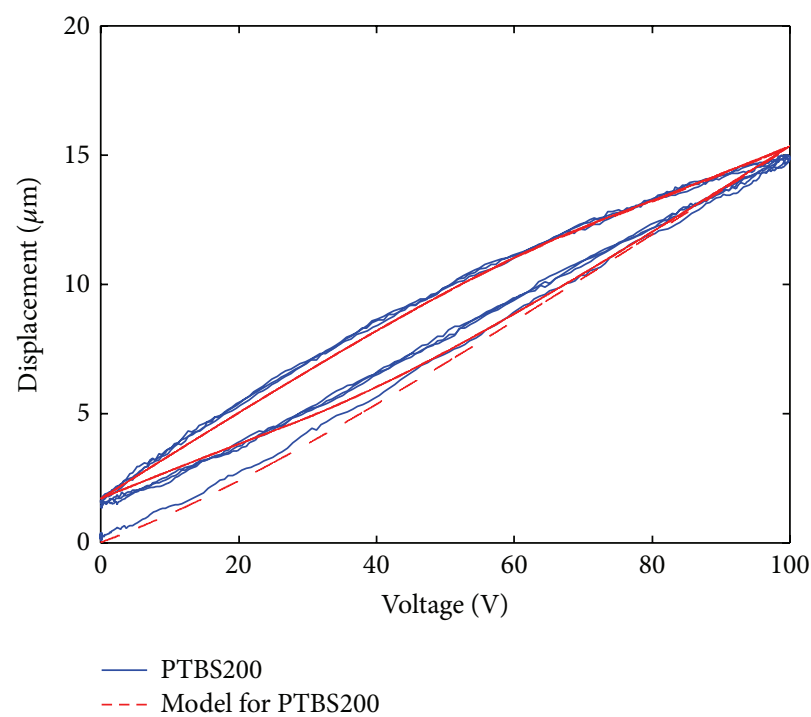

(a)

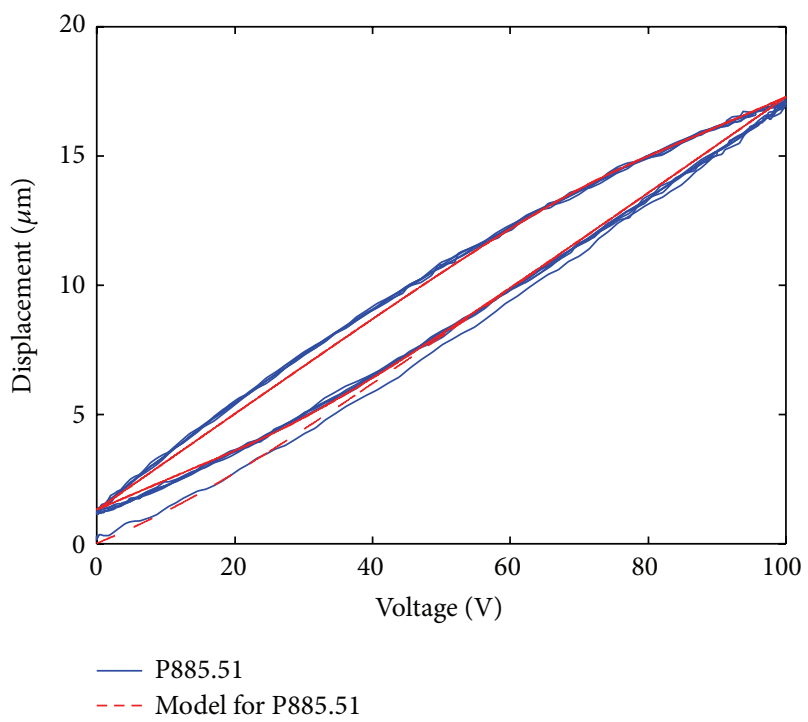

(c)

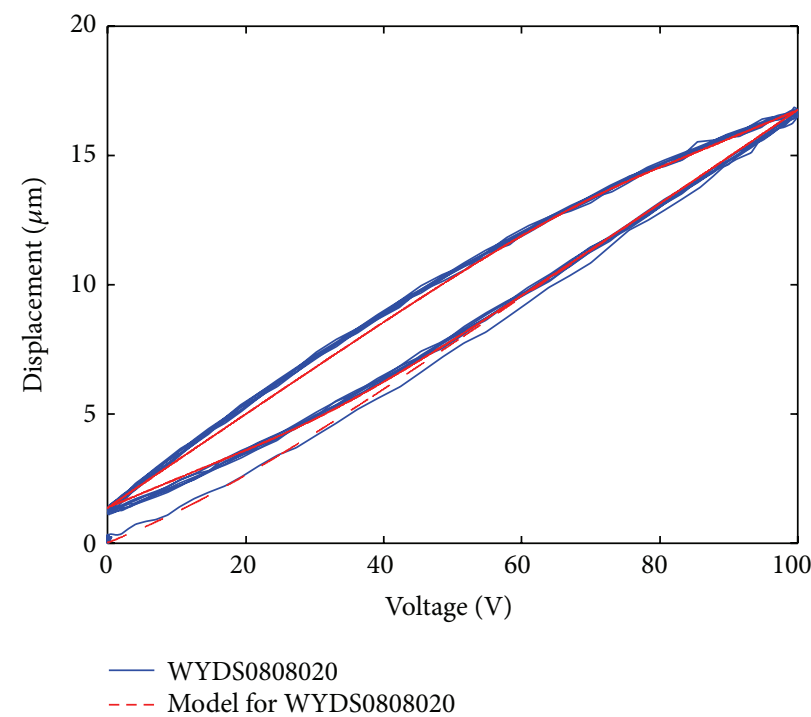

(b)

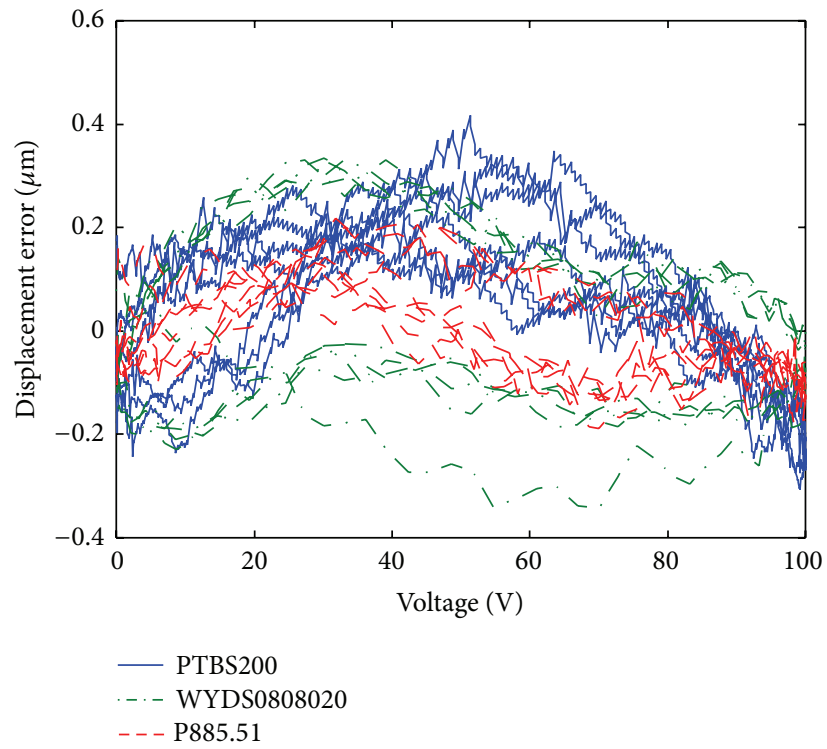

(d)

FIgURE 8: Hysteresis curves from the three PSAs measured by the LDV and predicted by the modified comprehensive model: (a) PTBS200, (b) WYDS0808020, (c) P885.51, and (d) the modeling errors.

sensors, the hysteresis observer is established to estimate the hysteresis force. The hysteresis observer can be expressed as

$$
\begin{aligned}
\hat{f}_{H}= & A \dot{u}-\beta|\dot{u}|\left|\widehat{f}_{H}\right|^{n-1} \widehat{f}_{H}-\gamma \dot{u}\left|\widehat{f}_{H}\right|^{n} \\
& +\delta u \operatorname{sgn}\left(\widehat{f}_{H}\right),
\end{aligned}
$$

where $\widehat{f}_{H}$ is the estimated value of $f_{H}$.

All coefficients in (37) are constant over time. However, the hysteresis characteristics of PSAs possess time variability, and using the hysteresis observer given by (40) with constant coefficients to observe the hysteresis force of PSAs will result in observation errors. In order to accurately observe the hysteresis force, we can use the recursive least-squares method instead of the least-squares method to identify online the estimated values $\widehat{A}, \widehat{\delta}, \widehat{n}, \widehat{\beta}$, and $\widehat{\gamma}$, which can make the hysteresis observer variable. When utilizing the hysteresis observer with the time variability to observe the hysteresis force, the observation error can be decreased theoretically. In this way, (40) can be rewritten as

$$
\begin{aligned}
\hat{f}_{H}= & \widehat{A} \dot{u}-\widehat{\beta}|\dot{u}|\left|\widehat{f}_{H}\right|^{\hat{n}-1} \widehat{f}_{H}-\widehat{\gamma} \dot{u}\left|\widehat{f}_{H}\right|^{\hat{n}} \\
& +\widehat{\delta} u \operatorname{sgn}\left(\widehat{f}_{H}\right) .
\end{aligned}
$$

Utilizing the observed value of the hysteresis force to compensate for PSAs, the transfer function of PSAs can be expressed as

$$
\begin{aligned}
& \frac{x(s)}{u(s)}=\frac{k_{L}+\left(f_{H}(s)-\hat{f}_{H}(s)\right) / u(s)}{\tau s+1} \\
& \cdot \frac{\left(c_{B}+c_{P}\right) s+\left(k_{B}+k_{P}\right)}{\left(\left(m_{P} / 4\right) s^{2}+c_{B} s+k_{B}\right)\left(\left(m_{P} / 4\right) s^{2}+c_{P} s+k_{P}\right)} .
\end{aligned}
$$




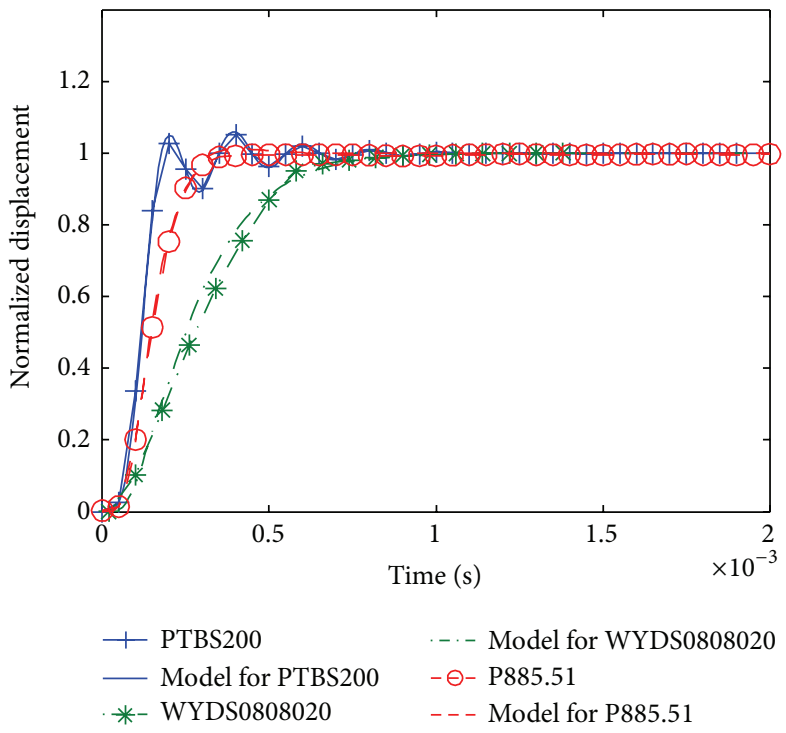

(a)

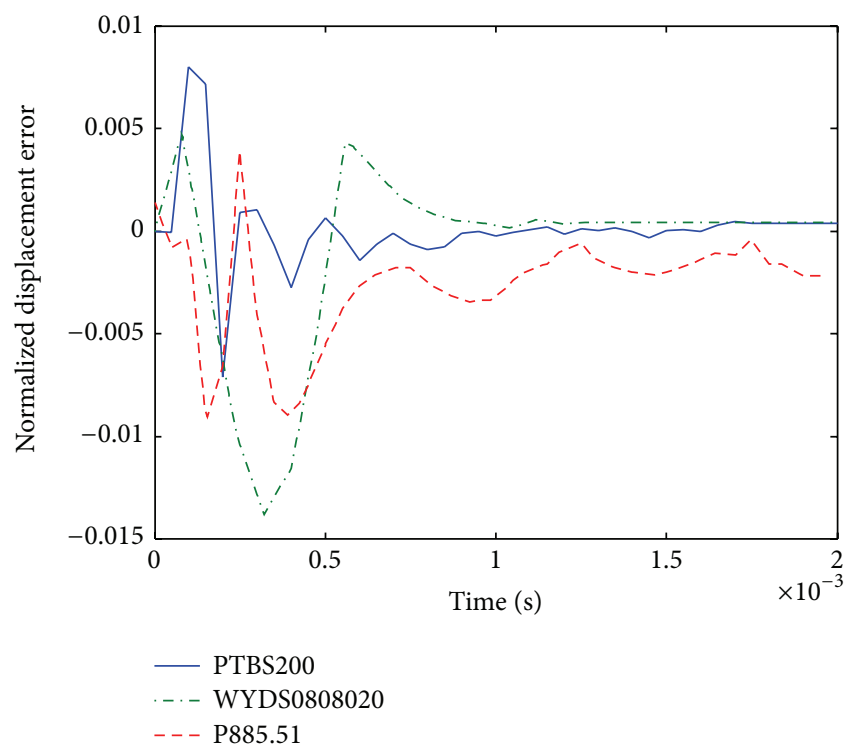

(b)

FIGURE 9: Normalized unit step responses of the three PSAs measured by the LDV and predicted by the modified comprehensive model: (a) the normalized unit step responses and (b) the modeling errors.

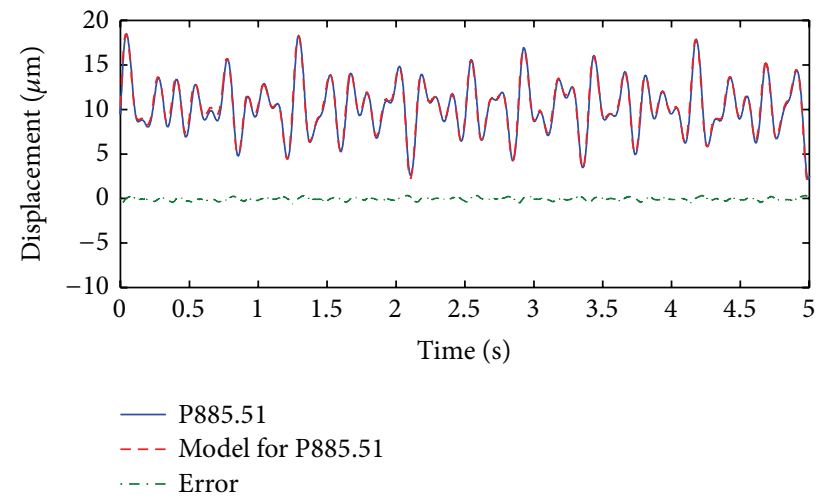

FIgURE 10: Time histories of the output displacements from P885.51 with nonperiodic voltage measured by the LDV and predicted by the modified comprehensive model and the modeling error.

When the observation error is small, (39) can be approximated as

$$
\begin{aligned}
& \frac{x(s)}{u(s)} \approx \frac{k_{L}}{\tau s+1} \\
& \cdot \frac{\left(c_{B}+c_{P}\right) s+\left(k_{B}+k_{P}\right)}{\left(\left(m_{P} / 4\right) s^{2}+c_{B} s+k_{B}\right)\left(\left(m_{P} / 4\right) s^{2}+c_{P} s+k_{P}\right)} .
\end{aligned}
$$

According to (39), the feedforward linearization control can linearize the PSAs to some extent. Moreover, when PSAs work in the quasi-static condition, (43) can be further approximated as

$$
\frac{x(s)}{u(s)} \approx k
$$

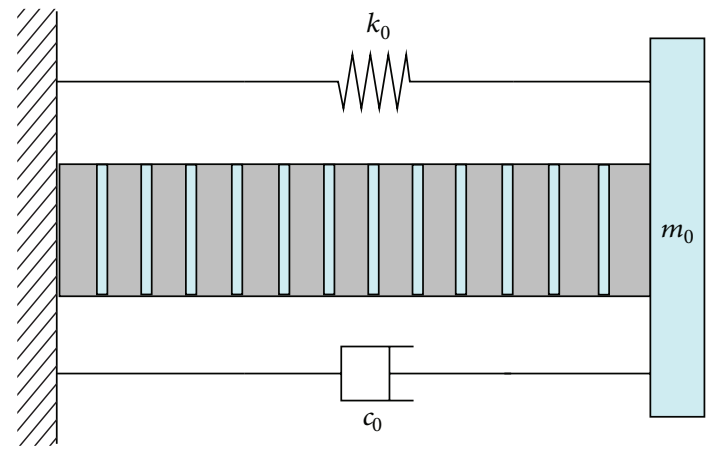

FIGURE 11: Schematic representation of prestressed PSAs.

where $k$ is defined by (23). Obviously, the linearization method can simplify the control algorithm and improve the control accuracy of PSAs.

The concrete realization method will be given in subsequent papers.

5.2. Design the Dynamic Characteristics. According to (10)(12), theoretically speaking, the dynamic performance of PSAs can be designed through configuring the one zero and two poles of the transfer function by controlling the characteristics of the bonding layers in the layering/stacking processes. However, it is difficult to precisely control the characteristics of the bonding layers. According to the proposed modified comprehensive model in this paper, the dynamic performance of PSAs can be simply and effectively designed through letting PSAs be prestressed with elastic deformation mechanisms, and the principle is shown in Figure 11. 


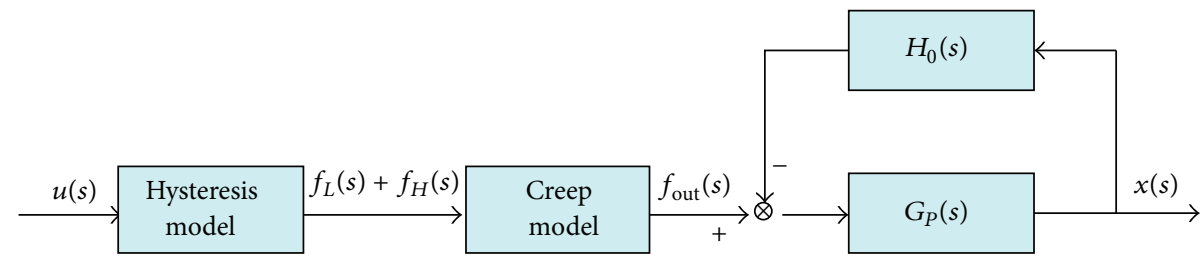

FIGURE 12: Block diagram of the comprehensive model for prestressed PSAs.

According to the proposed modified comprehensive model, the comprehensive model of the prestressed PSAs can be expressed as

$$
\frac{x(s)}{u(s)}=\frac{k_{L}+f_{H} / u(s)}{\tau s+1}\left[\frac{1}{\left(\left(m_{P} / 4\right) s^{2}+c_{B} s+k_{B}\right)\left(\left(m_{P} / 4\right) s^{2}+c_{P} s+k_{P}\right) /\left(\left(c_{B}+c_{P}\right) s+\left(k_{B}+k_{P}\right)\right)+\left(m_{0} s^{2}+c_{0} s+k_{0}\right)}\right],
$$

where $m_{0}, c_{0}$, and $k_{0}$ are the equivalent mass, damping, and stiffness of the prestressed mechanism, respectively. Let

$$
\begin{aligned}
& G_{P}(s) \\
& \quad=\frac{\left(c_{B}+c_{P}\right) s+\left(k_{B}+k_{P}\right)}{\left(\left(m_{P} / 4\right) s^{2}+c_{B} s+k_{B}\right)\left(\left(m_{P} / 4\right) s^{2}+c_{P} s+k_{P}\right)}, \\
& H_{0}(s)=m_{0} s^{2}+c_{0} s+k_{0} .
\end{aligned}
$$

According to (43) and (44), (42) can be rewritten as

$$
\frac{x(s)}{u(s)}=\frac{k_{L}+f_{H} / u(s)}{\tau s+1} \frac{G_{P}(s)}{1+G_{P}(s) H_{0}(s)} .
$$

According to (45), the stiffness, damping, and mass of the prestressed mechanism act as the position, velocity, and acceleration feedback, respectively. The block diagram of the comprehensive model for prestressed PSAs is shown in Figure 12. Therefore, the poles of the mechanical parts can be configured by choosing the values of the stiffness, damping, and mass of the prepressed mechanism; accordingly the dynamic performance of PSAs can be designed. It should be pointed out that, according to (45), the prepressed mechanism is unable to influence the creep characteristics of PSAs, but the creep characteristics can affect the dynamic performance of PSAs. Therefore, in order to more rationally design the dynamic performance, the impact of the creep characteristics on the dynamic performance of PSAs must be preconsidered.

\section{Conclusions}

Consider that a linear force and a hysteresis force will be generated by piezoelectric wafers under the applied voltage to a PSA, and the total force, which has suffered from creep, would result in the forced vibration of the 2-DOF massspring-damper system composed of the piezoelectric wafers and the bonding layers. A modified comprehensive model for PSAs was put forward by using a linear function, an asymmetrical Bouc-Wen hysteresis operator, and a creep function to model the linear force, the hysteresis force, and the creep characteristics, respectively. Through separating the linear component of the modified comprehensive model from the hysteresis component, the parameter identification method was established through identifying the parameters for the linear and hysteresis components separately by the limiting theorem of the Laplace transformation and the least-squares method, respectively. The experimental results showed that the modified comprehensive model for PSAs with the corresponding parameter identification method could accurately portray the hysteresis and dynamic characteristics of PSAs fabricated by different layering/stacking processes. Finally, the theoretical analyzing on utilizing the modified comprehensive model to linearize the hysteresis characteristics and design the dynamic characteristics of PSAs was given.

\section{Conflict of Interests}

The authors declare that there is no conflict of interests regarding the publication of this paper.

\section{Acknowledgments}

The authors wish to acknowledge the financial support by National Natural Science Foundation of China (Grant no. 61304137), the Scientific Research Foundation of Nanjing University of Posts and Telecommunications (Grant no. NY213111), the Key University Science Research Project of Jiangsu Province (Grant no. 15KJB130005), and the Fundamental Research Funds for the Central Universities (Grant no. 30915011326). 


\section{References}

[1] S. Devasia, E. Eleftheriou, and S. O. R. Moheimani, "A survey of control issues in nanopositioning," IEEE Transactions on Control Systems Technology, vol. 15, no. 5, pp. 802-823, 2007.

[2] W. Dong, J. Tang, and Y. ElDeeb, "Design of a linear-motion dual-stage actuation system for precision control," Smart Materials and Structures, vol. 18, no. 9, Article ID 095035, 11 pages, 2009.

[3] R. Ben Mrad and H. Hu, "A model for voltage-to-displacement dynamics in piezoceramic actuators subject to dynamic-voltage excitations," IEEE/ASME Transactions on Mechatronics, vol. 7, no. 4, pp. 479-489, 2002.

[4] P. Ge and M. Jouaneh, "Modeling hysteresis in piezoceramic actuators," Precision Engineering, vol. 17, no. 3, pp. 211-221, 1995.

[5] G. Song, J. Q. Zhao, X. Q. Zhou, and J. A. de Abreu-García, "Tracking control of a piezoceramic actuator with hysteresis compensation using inverse Preisach model," IEEE/ASME Transactions on Mechatronics, vol. 10, no. 2, pp. 198-209, 2005.

[6] Y. L. Zhang, Y. Zhang, C. H. Ru, B. K. Chen, and Y. Sun, "A load-lock-compatible nanomanipulation system for scanning electron microscope," IEEE/ASME Transactions on Mechatronics, vol. 18, no. 1, pp. 230-237, 2013.

[7] P. Mercorelli and N. Werner, "A hybrid actuator modelling and hysteresis effect identification in camless internal combustion engines control," International Journal of Modelling, Identification and Control, vol. 21, no. 3, pp. 253-263, 2014.

[8] Y. Qin, Y. Tian, D. Zhang, B. Shirinzadeh, and S. Fatikow, "A novel direct inverse modeling approach for hysteresis compensation of piezoelectric actuator in feedforward applications," IEEE/ASME Transactions on Mechatronics, vol. 18, no. 3, pp. 981989, 2013.

[9] U. X. Tan, W. T. Latt, C. Y. Shee, C. N. Riviere, and W. T. Ang, "Feedforward controller of ill-conditioned hysteresis using singularity-free prandtl-ishlinskii model," IEEE/ASME Transactions on Mechatronics, vol. 14, no. 5, pp. 598-605, 2009.

[10] S. Chonan, Z. Jiang, and T. Yamamoto, "Nonlinear hysteresis compensation of piezoelectric ceramic actuators," Journal of Intelligent Material Systems and Structures, vol. 7, no. 2, pp. 150156, 1996.

[11] L. Sun, C. Ru, W. Rong, L. Chen, and M. Kong, "Tracking control of piezoelectric actuator based on a new mathematical model," Journal of Micromechanics and Microengineering, vol. 14, no. 11, pp. 1439-1444, 2004.

[12] X. L. Zhang, Y. H. Tan, M. Y. Su, and Y. Q. Xie, "Neural networks based identification and compensation of rate-dependent hysteresis in piezoelectric actuators," Physica B: Condensed Matter, vol. 405, no. 12, pp. 2687-2693, 2010.

[13] J. P. Lien, A. York, T. Fang, and G. D. Buckner, "Modeling piezoelectric actuators with Hysteretic Recurrent Neural Networks," Sensors and Actuators A: Physical, vol. 163, no. 2, pp. 516-525, 2010.

[14] M. Goldfarb and N. Celanovic, "Modeling piezoelectric stack actuators for control of micromanipulation," IEEE Control Systems Magazine, vol. 17, no. 3, pp. 69-79, 1997.

[15] S.-H. Lee and T. J. Royston, "Modeling piezoceramic transducer hysteresis in the structural vibration control problem," Journal of the Acoustical Society of America, vol. 108, no. 6, pp. 28432855, 2000.

[16] M. Kamlah and Q. Jiang, "Constitutive model for ferroelectric PZT ceramics under uniaxial loading," Smart Materials and Structures, vol. 8, no. 4, pp. 441-459, 1999.
[17] J. W. Li, X. B. Chen, Q. An, S. D. Tu, and W. J. Zhang, "Friction models incorporating thermal effects in highly precision actuators," Review of Scientific Instruments, vol. 80, no. 4, Article ID 045104, 6 pages, 2009.

[18] S.-J. Huang and C.-M. Chiu, "Optimal LuGre friction model identification based on genetic algorithm and sliding mode control of a piezoelectric-actuating table," Transactions of the Institute of Measurement and Control, vol. 31, no. 2, pp. 181-203, 2009.

[19] D. H. Wang, W. Zhu, and Q. Yang, "Linearization of stack piezoelectric ceramic actuators based on Bouc-Wen model," Journal of Intelligent Material Systems and Structures, vol. 22, no. 5, pp. 405-417, 2011.

[20] D. H. Wang and W. Zhu, "A phenomenological model for prestressed piezoelectric ceramic stack actuators," Smart Materials and Structures, vol. 20, no. 3, Article ID 035018, 2011.

[21] O. Gomis-Bellmunt, F. Ikhouane, P. Castell-Vilanova, and J. Bergas-Jané, "Modeling and validation of a piezoelectric actuator," Electrical Engineering, vol. 89, no. 8, pp. 629-638, 2007.

[22] H. J. M. T. A. Adriaens, W. L. de Koning, and R. Banning, "Modeling piezoelectric actuators," IEEE/ASME Transactions on Mechatronics, vol. 5, no. 4, pp. 331-341, 2000.

[23] M. Quant, H. Elizalde, A. Flores, R. Ramírez, P. Orta, and G. Song, "A comprehensive model for piezoceramic actuators: modelling, validation and application," Smart Materials and Structures, vol. 18, no. 12, Article ID 125011, 16 pages, 2009. 

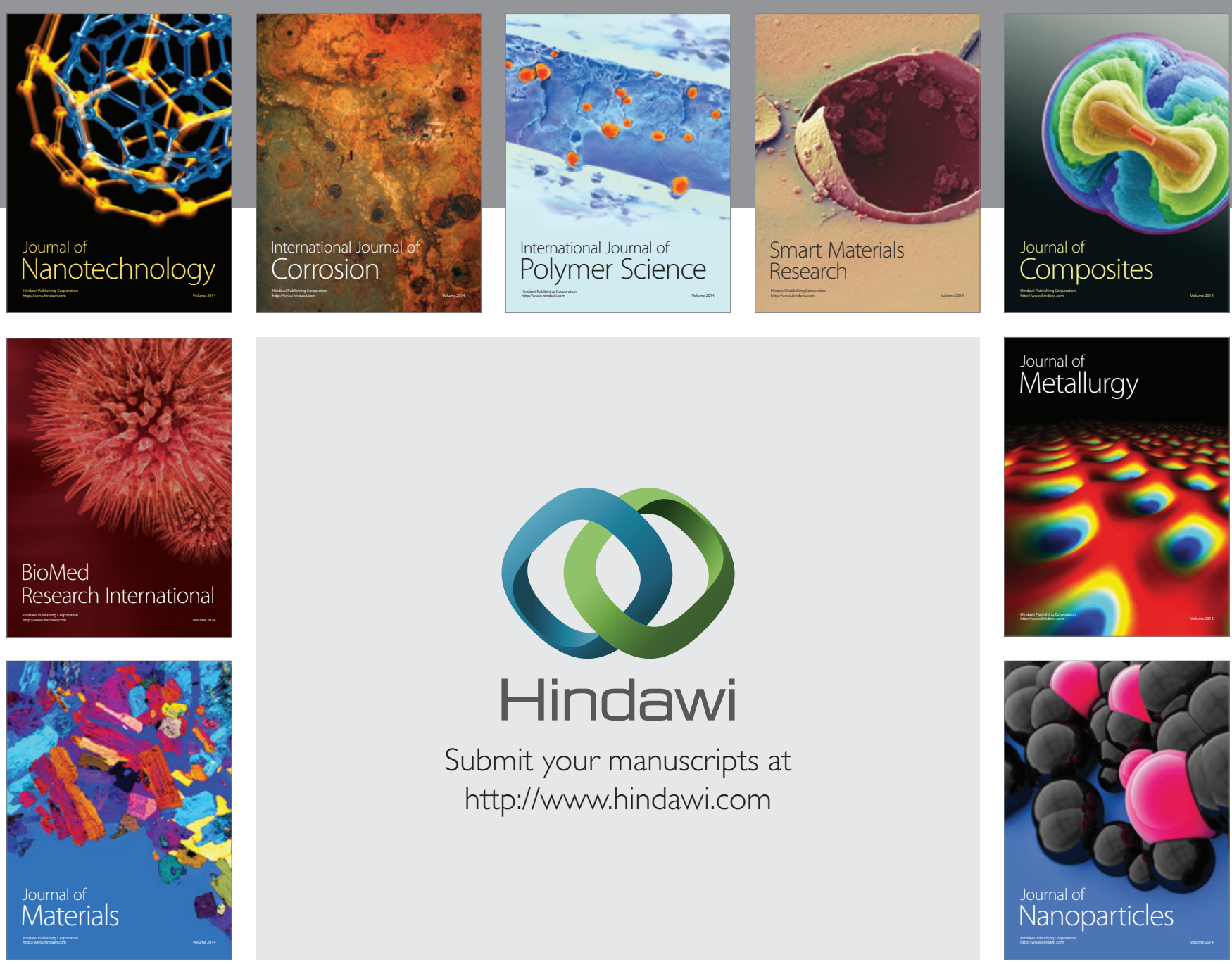

Submit your manuscripts at http://www.hindawi.com
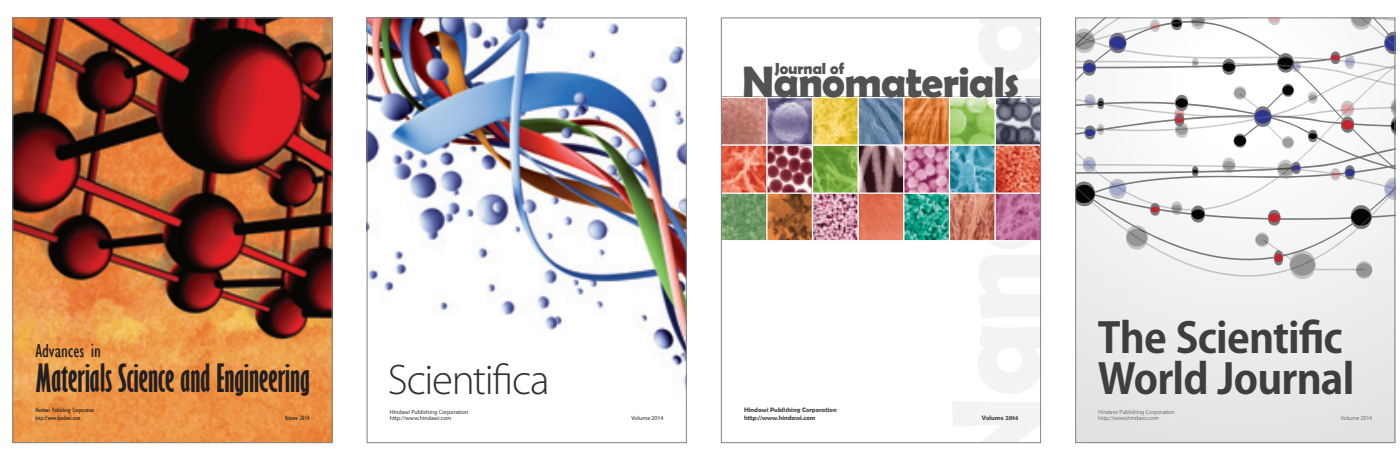

\section{The Scientific World Journal}
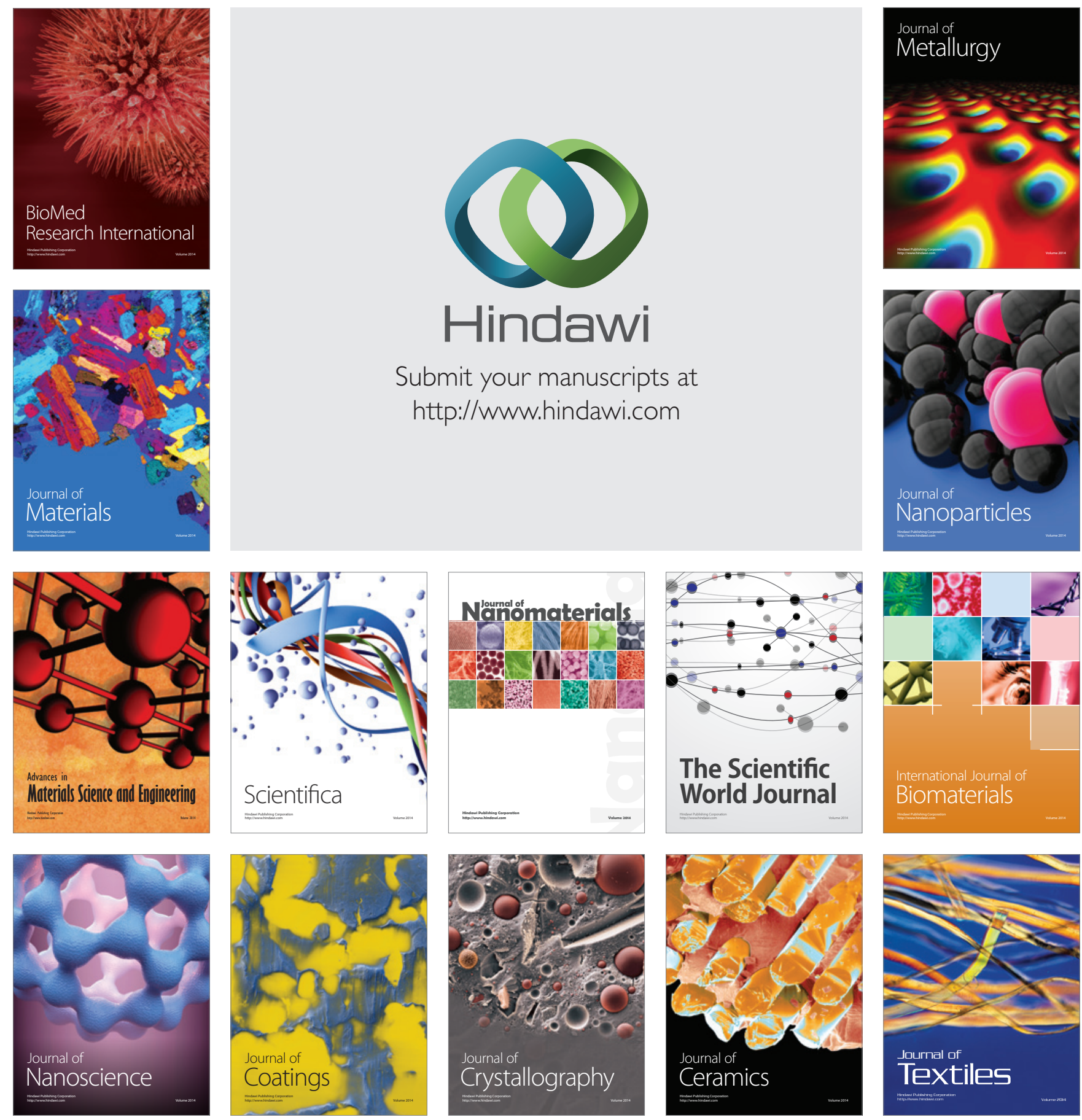